\title{
Institutional volume and the effect of recipient risk on short-term mortality after orthotopic heart transplant
}

\author{
George J. Arnaoutakis, MD, ${ }^{a}$ Timothy J. George, MD, ${ }^{a}$ Jeremiah G. Allen, MD, ${ }^{a}$ Stuart D. Russell, MD, ${ }^{b}$ \\ Ashish S. Shah, MD, ${ }^{\mathrm{a}}$ John V. Conte, MD, ${ }^{\mathrm{a}}$ and Eric S. Weiss, MD, MPH ${ }^{\mathrm{a}, \mathrm{c}}$
}

Objective: We developed a validated 50-point recipient risk index predicting short-term mortality after orthotopic heart transplant (OHT). This study examined the relationship between institutional volume and recipient risk on post-OHT mortality.

\begin{abstract}
Methods: We used United Network for Organ Sharing (UNOS) data to identify primary OHT recipients between January 2000 and April 2010. Centers were stratified by mean annual volume. Preoperative Index for Mortality Prediction After Cardiac Transplantation risk scores were calculated for each patient with our validated 50-point system. Primary outcomes were 30-day and 1-year survivals. Multivariable logistic regression analysis included interaction terms to examine effect modification of risk and volume on mortality.
\end{abstract}

Results: In all, 18,226 patients underwent transplant at 141 centers: $1173(6.4 \%)$ recipients at low-volume centers $(<7$ procedures/y), $5353(29.4 \%)$ at medium-volume centers $(7-15$ procedures/y), and 11,700 $(64.2 \%)$ at high-volume centers $(>15$ procedures/y). Low center volume was associated with worse 1-year mortality (odds ratio, 1.58; 95\% confidence interval, 1.30-1.92; $P<.001$ ). For 1 -year survival, there was significant positive interaction between center volume and recipient risk score (odds ratio, 1.04; $95 \%$ confidence interval, 1.01-1.07; $P=.02$ ), indicating effect of risk on mortality at low-volume centers greater than from either variable analyzed individually. Among high-risk recipients (score $\geq 10$ ), 1-year survival was improved at high-volume centers (high, 79\%; medium, 75\%; low, 64\%).

Conclusions: In analysis of UNOS data with our validated recipient risk index, institutional volume acted as an effect modifier on association between risk and mortality. High-risk patients had higher mortality at low-volume centers; differences dissipated among lower-risk recipients. These data support a mandate for high-risk transplants at higher-volume centers. (J Thorac Cardiovasc Surg 2012;143:157-67)

\section{Supplemental material is available online.}

From the Division of Cardiac Surgery, ${ }^{\text {a }}$ Department of Surgery, the Division of Cardiology, ${ }^{b}$ Department of Medicine, and the Bloomberg School of Public Health, ${ }^{c}$ The Johns Hopkins Medical Institutions, Baltimore, Md.

G.J.A. and E.S.W. are Irene Piccinini Investigators in Cardiac Surgery. J.G.A. and T.J.G. are Hugh R. Sharp Cardiac Surgery Research Fellows. This research was supported in part by National Institutes of Health grant 1T32CA126607-01A2 (to G.J.A.) and also supported by Health Resources and Services Administration contract 231-00-0115.

Disclosures: J.V.C. receives research support from Medtronic (Medtronic, Inc, Minneapolis, Minn) and Thoratec (Thoratec Corporation, Pleasanton, Calif). The content is the responsibility of the authors alone and does not necessarily reflect the views or policies of the Department of Health and Human Services, nor does mention of trade names, commercial products, or organizations imply endorsement by the US government

Read at the 91st Annual Meeting of The American Association for Thoracic Surgery, Philadelphia, Pennsylvania, May 7-11, 2011.

Received for publication May 6, 2011; revisions received Aug 17, 2011; accepted for publication Sept 26, 2011.

Address for reprints: Eric S. Weiss, MD, MPH, Division of Cardiothoracic Surgery, Department of Surgery, New York Presbyterian Hospital, Columbia University College of Physicians and Surgeons, 177 Fort Washington Ave, MGH 7GN-435, New York, NY 10032 (E-mail: esw9006@ columbia.edu).

$0022-5223 / \$ 36.00$

Copyright (C) 2012 Published by Elsevier Inc. on behalf of The American Association for Thoracic Surgery

doi:10.1016/j.jtcvs.2011.09.040
More than 30 years have elapsed since the early observation that outcomes for a broad array of surgical procedures improve with increasing hospital center volume. ${ }^{1}$ Many subsequent studies have documented this relationship for a spectrum of surgical procedures. ${ }^{2-10}$ Specifically, in the field of solid organ transplantation, volume-outcome associations have been demonstrated in population-based studies for kidney, liver, lung, and heart transplants. ${ }^{11-15}$ In the absence of prospective data, these previous studies could only identify associations. These associations likely represent the different systems or processes of care among centers with different levels of procedural volume, which are difficult to measure objectively.

It is possible that certain centers may only have the institutional experience and support framework to treat patients within a given risk profile. Recipient risk and center volume have been examined in orthotopic heart transplant (OHT) recipients; however, the assignment of recipient risk was not fully characterized. ${ }^{14}$ We recently developed a novel, quantitative recipient risk index for mortality prediction after cardiac transplant, the Index for Mortality Prediction After Cardiac Transplantation (IMPACT), from the data in the United Network for Organ Sharing (UNOS) registry. ${ }^{16}$ The effect of center volume was not included in the original IMPACT study, because the aim of that initial study was to 


\section{Abbreviations and Acronyms \\ $\mathrm{CI}=$ confidence interval \\ IMPACT $=$ Index for Mortality Prediction After Cardiac Transplantation \\ OHT = orthotopic heart transplant \\ OR = odds ratio \\ UNOS = United Network for Organ Sharing}

define a recipient risk index that was broadly applicable on the basis of only recipient characteristics. In this study, we incorporated the IMPACT risk index to test the hypothesis that the effect of recipient risk on short-term mortality after OHT varies by different levels of center volume. This interaction, or effect modification, has not been previously studied for OHT. With a validated risk index, these findings may begin to explain the previously observed outcome differences associated with center volume.

\section{MATERIALS AND METHODS \\ Data Source}

UNOS provided Standard Transplant Analysis and Research files with donor-specific and follow-up data from October 1, 1987, to December 31,2010 . The registry consists of a prospectively collected database of unique demographic, donor, operative, and postoperative information for all thoracic transplant recipients in the United States. No patient or center identifiers were included, and this study was granted exemption status by the institutional review board.

\section{Study Design}

This was a retrospective review involving all primary, adult ( $\geq 18$ years) OHT recipients from January 2000 to April 2010, to ensure adequate time for follow-up. All patients who received simultaneous liver transplants $(n=69)$, and those with total artificial hearts $(n=71)$ were excluded. All patients included in the analysis were assigned the appropriate IMPACT risk score. ${ }^{13}$ The IMPACT risk score is a quantitative risk index derived from and cross-validated with UNOS data, and it has been shown to be highly predictive of 1-year mortality for adult patients undergoing firsttime OHT. ${ }^{16}$ The risk index incorporates 12 recipient-specific preoperative variables (age, sex, ethnicity, diagnosis, creatinine clearance, preoperative dialysis, serum bilirubin, preoperative infection, need for mechanical ventilation, intra-aortic balloon pump, temporary circulatory support, and ventricular assist device) to assign relative points out of a possible maximum of 50 (Table E1). No patients were missing the data necessary to compose their respective scores.

\section{Center Volume}

Center code was provided as a random encrypted alphanumeric 6-character code unique to each individual center. Center and patient level data were deidentified in this data set. The variable of average annual center volume for each center was derived from existing data. Accordingly, each patient outcome was linked with the corresponding hospital volume calculation. Years in which a center was clearly not performing OHT procedures were subtracted for purposes of average annual volume determination. Annual center volume was then ranked in thirds, with each center counting as an individual observation, and categorized as low ( $\leq 7$ OHT procedures/y), medium ( $>7$ to $\leq 15$ OHT procedures/y), or high ( $>15$ OHT procedures/y).
Categorization in this fashion prevented skewing the cut points upward because of a greater overall number of patients undergoing OHT at higher-volume centers. For ease of interpreting regression coefficients, center volume was reported as a categoric variable in regression analysis; however, we also examined center volume as a continuous variable to aid in identifying inflection points regarding risk of 1 -year mortality.

\section{Analysis}

The primary outcomes of interest were all-cause 30-day and 1-year mortalities. Additional postoperative complications examined were drug-treated rejection in the first year after transplant, postoperative renal replacement therapy, and need for cardiac reoperation. Multivariable logistic regression analysis incorporated recipient risk (IMPACT) score, allograft ischemic time, donor age, and annual center volume (low, medium, or high). Allograft ischemic time and center volume were examined as both continuous and categoric variables with cutoffs with the Akaike information criterion used to identify models with highest explanatory power in a nested model approach. According to the Akaike information criterion, the multivariable regression models using these 2 variables with cut points as categoric variables increased the explanatory power of the models. Separate regressions were performed for 30-day mortality and 1-year mortality. The Pearson $\chi^{2}$ goodness-of-fit test and area under the receiver operating characteristic curves were used to confirm the explanatory power of the regression models.

To test for an effect modification of center volume on recipient risk and short-term mortality, an interaction term was constructed. This interaction term consisted of the product of recipient risk score and the inverse of annual center volume. In this way, risk estimates of the individual components of the interaction term would be unidirectional, facilitating interpretation of the coefficients. When added to the multivariable regression models, this interaction term allowed the odds of early mortality to vary by level of center volume.

One-year survival was modeled according to the Kaplan-Meier method, with censoring for patients unavailable for follow-up or for administrative purposes (alive at the end of the study). A subgroup survival analysis was performed among patients in the highest quartile of risk (IMPACT score $>10$ ). Means are displayed with SDs, medians with interquartile ranges, and odds ratios (ORs) with 95\% confidence intervals (CIs). Biostatistical analysis was performed with STATA software (version 11 SE; StataCorp LP, College Station, Tex).

\section{RESULTS}

\section{Cohort Statistics}

There were 18,226 OHT recipients who satisfied inclusion criteria, and these transplants were performed at a total of 141 unique centers. The mean age was $52 \pm 12$ years, and $4311(23.7 \%)$ were women. Recipient ethnic distribution was as follows: $73.1 \%$ white $(\mathrm{n}=13,328), 16.4 \%$ African American $(\mathrm{n}=2,988), 7.2 \%$ Hispanic $(\mathrm{n}=1311)$, and $3.3 \%$ other $(\mathrm{n}=599)$. Donor ethnic distribution was as follows: $69.0 \%$ white $(\mathrm{n}=12,573), 13.2 \%$ African American $(\mathrm{n}=2405), 15.3 \%$ Hispanic $(\mathrm{n}=2793)$, and $2.5 \%$ other $(\mathrm{n}=455)$. During the study period, 4892 patients died (incidence 6.6 deaths/100 person-years). The Kaplan-Meier cumulative incidence of 1 -year mortality was $13.6 \%$ $(n=2223)$. Median follow-up was 45 months (interquartile range, 15-75 months).

\section{Center Volume}

Mean annual institutional volume ranged from 0.2 OHT procedures/y to 76 OHT procedures/y, with a median of 10 
TABLE 1. Patient characteristics and postoperative outcomes stratified by center volume

\begin{tabular}{|c|c|c|c|c|}
\hline \multirow[b]{2}{*}{ Variable } & \multicolumn{3}{|c|}{ Volume } & \multirow[b]{2}{*}{$P$ value* } \\
\hline & Low $(N=1173)$ & Medium $(N=\mathbf{5 3 5 3})$ & $\operatorname{High}(\mathbf{N}=11,700)$ & \\
\hline \multicolumn{5}{|l|}{ Demographics } \\
\hline IMPACT score $($ mean $\pm \mathrm{SD})$ & $6.3 \pm 4.0$ & $6.2 \pm 3.7$ & $6.2 \pm 3.8$ & .8 \\
\hline Age $(y$, mean $\pm S D)$ & $48.9 \pm 13.9 \dagger$ & $51.7 \pm 11.9 \dagger$ & $52.5 \pm 12.1$ & $<.01$ \\
\hline Female (no.) & $262(22.4 \%)$ & $1318(24.6 \%)$ & $2731(23.3 \%)$ & .1 \\
\hline White (no.) & $839(71.5 \%) \dagger$ & $3792(70.8 \%) \dagger$ & $8697(74.3 \%)$ & $<.01$ \\
\hline African American (no.) & $226(19.3 \%) \dagger$ & $952(17.8 \%) \dagger$ & $1810(15.5 \%)$ & $<.001$ \\
\hline Diagnosis (no.) & & & & $<.01$ \\
\hline Idiopathic & $452(38.5 \%)$ & $2288(42.7 \%)$ & $4988(42.6 \%)$ & \\
\hline Ischemic & $535(45.6 \%)$ & $2508(46.9 \%) \dagger$ & $5165(44.2 \%)$ & \\
\hline Congenital & $89(7.6 \%) \dagger$ & $95(1.8 \%) \dagger$ & $285(2.4 \%)$ & \\
\hline Other & $97(8.3 \%)$ & $462(8.6 \%)$ & $1262(10.8 \%)$ & \\
\hline \multicolumn{5}{|l|}{ Acuity } \\
\hline Hypertension (no.) & $373(39.7 \%)$ & $1593(44.0 \%) \dagger$ & $3200(39.0 \%)$ & $<.01$ \\
\hline Diabetes mellitus (no.) & $220(18.9 \%) \dagger$ & $1301(24.5 \%)$ & $2677(23.2 \%)$ & $<.01$ \\
\hline Creatinine clearance $\ddagger(\mathrm{mL} / \mathrm{min}$, mean $\pm \mathrm{SD})$ & $68.8 \pm 27.1 \dagger$ & $67.3 \pm 27.1 \dagger$ & $65.5 \pm 25.5$ & $<.01$ \\
\hline Serum bilirubin $(\mathrm{mg} / \mathrm{mL}$, mean $\pm \mathrm{SD})$ & $1.4 \pm 3.1 \dagger$ & $1.2 \pm 2.2$ & $1.3 \pm 2.3$ & .02 \\
\hline Preoperative mechanical ventilation (no.) & $34(2.9 \%)$ & $172(3.2 \%) \dagger$ & $277(2.4 \%)$ & $<.01$ \\
\hline Ischemic time $(\mathrm{h}$, mean $\pm \mathrm{SD})$ & $3.0 \pm 1.1 \dagger$ & $3.1 \pm 1.0 \dagger$ & $3.3 \pm 1.0$ & $<.01$ \\
\hline Temporary circulatory support $\S$ (no.) & $26(2.2 \%) \dagger$ & $62(1.2 \%)$ & $165(1.4 \%)$ & .1 \\
\hline \multicolumn{5}{|l|}{ Ventricular assist device } \\
\hline Early generation $\|$ (no.) & $188(16.0 \%)$ & $891(16.6 \%) \dagger$ & $1724(14.7 \%)$ & $<.01$ \\
\hline Late generation $\Phi($ no.) & $5(0.4 \%)$ & $34(0.6 \%)$ & $45(0.4 \%)$ & .08 \\
\hline HeartMate II (no.) & $24(2.1 \%) \ddagger$ & $307(5.7 \%) \dagger$ & $571(4.9 \%)$ & $<.01$ \\
\hline Intra-aortic balloon pump (no.) & $43(3.7 \%) \dagger$ & $267(5.0 \%)$ & $656(5.6 \%)$ & $<.01$ \\
\hline \multicolumn{5}{|l|}{ Postoperative outcomes } \\
\hline Need for cardiac reoperation (no.) & $120(13.0 \%)$ & $409(11.7 \%)$ & $881(11.6 \%)$ & .4 \\
\hline Stroke (no.) & $26(2.2 \%)$ & $140(2.6 \%)$ & $250(2.2 \%)$ & .2 \\
\hline New-onset dialysis (no.) & $113(9.9 \%) \dagger$ & $423(8.1 \%) \dagger$ & $799(7.2 \%)$ & $<.01$ \\
\hline Treated rejection in first year (no.) & $355(40.3 \%) \dagger$ & $1457(34.4 \%) \dagger$ & $2598(28.8 \%)$ & $<.01$ \\
\hline
\end{tabular}

IMPACT, Index for Mortality Prediction After Cardiac Transplantation. $* P$ value based on analysis of variance (continuous variables) or $\chi^{2}$ test (categoric variables). $\dagger$ Post hoc pairwise comparison $P<.05$ (reference high-volume centers) by Tukey-Kramer method for continuous variables or univariate logistic regression for categoric variables. $\ddagger$ Based on Cockcroft-Gault calculation. ${ }^{5}$ Includes extracorporeal membrane oxygenation or extracorporeal ventricular assist devices: Abiomed BVS5000 (Abiomed, Inc, Danvers, Mass), Bio-Medicus (Medtronic, Inc, Minneapolis, Minn), TandemHeart (Cardiac Assist, Inc, Pittsburgh, Pa), and Levitronix/Centrimag (Levitronix, Waltham, Mass). ||Early generation includes paracorporeal and intracorporeal pulsatile ventricular assist devices: Abiomed AB5000, HeartMate I (Thoratec Corporation, Pleasanton, Calif), XE and XVE, ThortecIVAD (Thoratec), Toyobo (Toyobo Co, Ltd, Osaka, Japan), Novacor (World Heart Inc, Oakland, Calif), Medos (Medos Medizintechnik AG, Stolberg, Germany), and LionHeart (Arrow International Inc, Reading Pa). 9 Later-generation continuous ventricular assist devices including Jarvik (Jarvik Heart, Inc, New York, NY), MicroMed Debakey (MicroMed Cardiovascular, Inc, Houston, Texas), and VentrAssist (Ventracor, Sydney, Australia), excluding HeartMate II.

OHT procedures/y. Primary stratification revealed that 173 patients $(6.4 \%)$ underwent transplant at centers with low volume ( $\leq 7$ OHT procedures/y, $\mathrm{n}=47), 5353$ patients $(29.4 \%)$ at centers with medium volumes $(>7$ to $\leq 15$ OHT procedures/y, $\mathrm{n}=47)$, and $11,700(64.2 \%)$ at centers with high volumes $(>15$ OHT procedures/y; $\mathrm{n}=47)$. Seventy-four centers $(52.4 \%)$ performed more than 10 OHT procedures/y, and 28 centers $(19.8 \%)$ performed more than 20 OHT procedures/y.

\section{IMPACT Score Generation}

After assigning all patients their respective risk score index values, the average score for the entire cohort was 6.24 \pm 3.77 . A histogram and test for skewness revealed that the pattern of scores followed a normal distribution, with values ranging from 0 to 33 . When divided into quartiles, the breakdown of scores was as follows: quartile 1,0 to less than $3(\mathrm{n}=4528 ; 24.8 \%)$; quartile 2,3 to less than $6(\mathrm{n}=3908 ; 21.4 \%)$; quartile 3,6 to less than 9 $(\mathrm{n}=5760 ; 31.6 \%)$; and quartile 4 , at least $9(\mathrm{n}=4030$; $22.1 \%)$. There were 2129 patients $(11.6 \%)$ with a risk score greater than 10 and only $92(0.5 \%)$ with a risk score greater than 20 .

\section{Baseline Characteristics}

Baseline characteristics were compared among the 3 center volume groups (Table 1). For post hoc comparisons the high-volume group served as the reference. Although there were several differences in baseline characteristics, patients were evenly matched among the 3 groups when examining overall recipient risk as assessed by the IMPACT risk score $(P=.8)$. Patients at high-volume centers tended to be white 

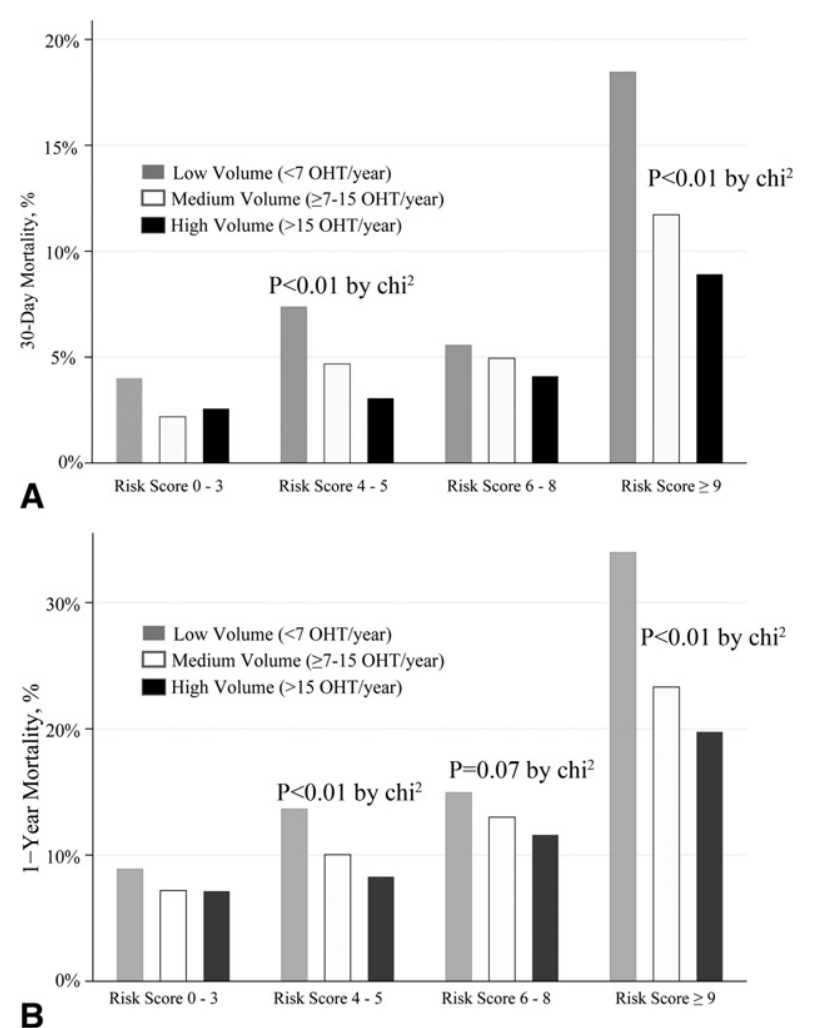

FIGURE 1. A, Bar graph depicting actual 30-day mortality by center volume (low, medium, and high) according to increasing risk strata. $P$ values are nonsignificant except where explicitly noted on the plot region. B, Bar graph depicting actual 1-year mortality by center volume (low, medium, and high) according to increasing risk strata. $P$ values are nonsignificant except where explicitly noted on the plot region. $O H T$, Orthotopic heart transplant.

and older. The presence of diabetes mellitus, renal dysfunction, intra-aortic balloon pump, and prolonged ischemic time were also more common among recipients at highvolume centers. Patients at low-volume centers tended to have congenital cardiac disease, tended to have higher serum bilirubin levels, and were more likely to be African American. At medium-volume centers, there were more patients with ischemic cardiomyopathy, history of hypertension, need for mechanical ventilation, and use of earlygeneration ventricular assist devices.

\section{Mortality and Risk-Adjusted Regression Analysis}

At 30 days, the unadjusted observed mortalities were $4.6 \%$ (high-volume centers), 5.6\% (medium-volume centers), and $9.3 \%$ (low-volume centers). Observed 1-year mortalities, unadjusted for recipient risk, were $11.6 \%$ (high-volume centers), $13.5 \%$ (medium-volume) and 18.1\% (low-volume centers). Differences in observed mortality by center volume were more pronounced when examined across increasing risk strata (Figure 1), with significant differences at 30 days and 1 year. In the adjusted multivariable logistic regression analysis, each 1-point increase in recipient risk score corresponded to a $14 \%$ increase in the odds of 1-year mortality (OR, 1.14; 95\% CI, 1.13-1.15; $P<.001$; Table 2). Increasing donor age (OR, $1.01 ; 95 \% \mathrm{CI}, 1.00-1.02 ; P<.001)$ and prolonged allograft ischemic time (OR, 1.75; 95\% CI, 1.42$2.15 ; P<.001)$ also increased the odds of 1-year mortality. For the 1-year mortality logistic regression model, the area under the receiver operating characteristic curve was 0.67, and the Pearson $\chi^{2}$ statistic was $5180(P=.25)$, indicating acceptable model fit.

When allowing the effect of recipient risk on 1-year mortality to vary by center volume, there was a positive and significant test of interaction (OR, 1.04; 95\% CI, $1.01-1.07 ; P<.001)$. This interaction signifies that highvolume centers minimized the effect of recipient risk, whereas low-volume centers amplified the odds of 1-year mortality associated with higher recipient risk. This effect modification is depicted graphically in Figure 2. As recipient risk scores increased, the difference in observed 1-year mortality for each tier of center volume became more pronounced, with observed mortality exceeding predicted mortality for low-volume and medium-volume centers. Conversely, the risk of death for high-volume centers was lower than would be predicted from the model, implying protection for high-risk patients at higher-volume centers. It is important to note that there were many low-volume centers that also achieved low 1-year mortality results, as shown in Figure 3. Because low-volume centers had a higher prevalence of congenital heart disease, the entire analysis was performed again without patients with congenital heart disease to ensure that this variable was not confounding the results. The magnitude and significance levels of the ORs for center volume, IMPACT risk score, and the interaction effect remained similar (test of interaction OR,1.06; 95\% CI, 1.02-1.10; $P=.004$ ).

Incorporating 4 quartiles of recipient risk according to the IMPACT risk score, relative risk of 1-year mortality is plotted against center volume as a continuous variable in Figure 4. As center volume increases from 0 to $10 \mathrm{OHT}$ procedures/y, there is a steep decline in risk of 1-year mortality for all 4 tiers of recipient risk, although the decline is least pronounced in the lowest-risk recipients. In the 2 lower quartiles of recipient risk, the relative risk of 1-year mortality crosses a threshold of 1 at a center volume of approximately 10 OHT procedures/y. In contrast, for patients in the upper 2 quartiles according to IMPACT risk score, the inflection point for relative risk of 1-year mortality occurs at a center volume of approximately $35 \mathrm{OHT}$ procedures/y, with an observed plateau beyond 35 to 40 OHT procedures/y. Consistent with this relative ratio plot, when center volume was examined as a continuous variable in the regression, increasing center volume was associated with lower odds of 1-year mortality (OR, 0.99; 95\% CI, 0.98-0.99; $P<.01)$. 
TABLE 2. Odds ratio estimates for 30-day and 1-year mortalities

\begin{tabular}{|c|c|c|c|c|c|c|}
\hline \multirow[b]{2}{*}{ Variable } & \multicolumn{3}{|c|}{ 30-d } & \multicolumn{3}{|c|}{$1 \mathbf{y}$} \\
\hline & OR & 95\% CI & $P$ value & OR & $\mathbf{9 5} \%$ CI & $P$ value \\
\hline \multicolumn{7}{|l|}{ Without volume-risk interaction effect } \\
\hline IMPACT score & 1.15 & $1.14-1.17$ & $<.001$ & 1.14 & $1.13-1.15$ & $<.001$ \\
\hline \multicolumn{7}{|l|}{ Center volume } \\
\hline High & Reference & - & - & Reference & - & - \\
\hline Medium & 1.28 & $1.10-1.49$ & .001 & 1.22 & $1.10-1.35$ & $<.001$ \\
\hline Low & 2.25 & $1.80-2.82$ & $<.001$ & 1.76 & $1.50-2.09$ & $<.001$ \\
\hline \multicolumn{7}{|l|}{ Allograft ischemic time } \\
\hline$<2 \mathrm{~h}$ & Reference & - & - & Reference & - & - \\
\hline$\geq 2 \mathrm{~h}$ to $<4 \mathrm{~h}$ & 1.20 & $0.93-1.54$ & .2 & 1.06 & $0.90-1.25$ & .4 \\
\hline$\geq 4 \mathrm{~h}$ to $<6 \mathrm{~h}$ & 1.91 & $1.46-2.49$ & $<.001$ & 1.58 & $1.33-1.89$ & $<.001$ \\
\hline$>6 \mathrm{~h}$ & 2.54 & $1.88-3.43$ & $<.001$ & 1.75 & $1.42-2.15$ & $<.001$ \\
\hline Donor age & 1.01 & $1.01-1.02$ & $<.001$ & 1.01 & $1.01-1.02$ & $<.001$ \\
\hline \multicolumn{7}{|l|}{ With volume-risk interaction effect } \\
\hline IMPACT score & 1.15 & $1.13-1.16$ & $<.001$ & 1.13 & $1.12-1.15$ & $<.001$ \\
\hline \multicolumn{7}{|l|}{ Center volume } \\
\hline High & Reference & - & - & Reference & - & - \\
\hline Medium & 1.29 & $1.12-1.49$ & .001 & 1.20 & $1.09-1.33$ & $<.001$ \\
\hline Low & 1.86 & $1.46-2.38$ & $<.001$ & 1.58 & $1.30-1.92$ & $<.001$ \\
\hline Center volume-risk interaction effect & 1.03 & $1.01-1.06$ & .04 & 1.04 & $1.01-1.07$ & .02 \\
\hline \multicolumn{7}{|l|}{ Allograft ischemic time } \\
\hline$<2 \mathrm{~h}$ & Reference & - & - & Reference & - & - \\
\hline$\geq 2 \mathrm{~h}$ to $<4 \mathrm{~h}$ & 1.20 & $0.93-1.55$ & .2 & 1.06 & $0.91-1.25$ & .4 \\
\hline$\geq 4 \mathrm{~h}$ to $<6 \mathrm{~h}$ & 1.92 & $1.46-2.51$ & $<.001$ & 1.59 & $1.33-1.89$ & $<.001$ \\
\hline$>6 \mathrm{~h}$ & 2.51 & $1.86-3.39$ & $<.001$ & 1.75 & $1.42-2.15$ & $<.001$ \\
\hline Donor age & 1.01 & $1.01-1.02$ & $<.001$ & 1.01 & $1.01-1.02$ & $<.001$ \\
\hline
\end{tabular}

IMPACT, Index for Mortality Predication After Cardiac Transplantation; $O R$, odds ratio; $C I$, confidence interval.

\section{Survival}

When comparing survival by center volume with the CoxMantel log-rank test, there was improved 1-year survival at high-volume centers $(P<.001$; Figure 5, A). Post hoc comparisons revealed a significant difference between high-volume and medium-volume centers for the cohort overall $(P=.04)$. High-risk patients only (IMPACT score $\geq 10$ ) were examined in a subgroup analysis, and the



FIGURE 2. Best-fit Lowess smoothed line for observed 1-year mortality by level of center volume (low, medium, and high) plotted against predicted 1-year mortality according to Index for Mortality Prediction After Cardiac Transplantation (IMPACT) score. The 95\% confidence interval (CI) for predicted mortality is in the shaded area. OHT, Orthotopic heart transplant. 


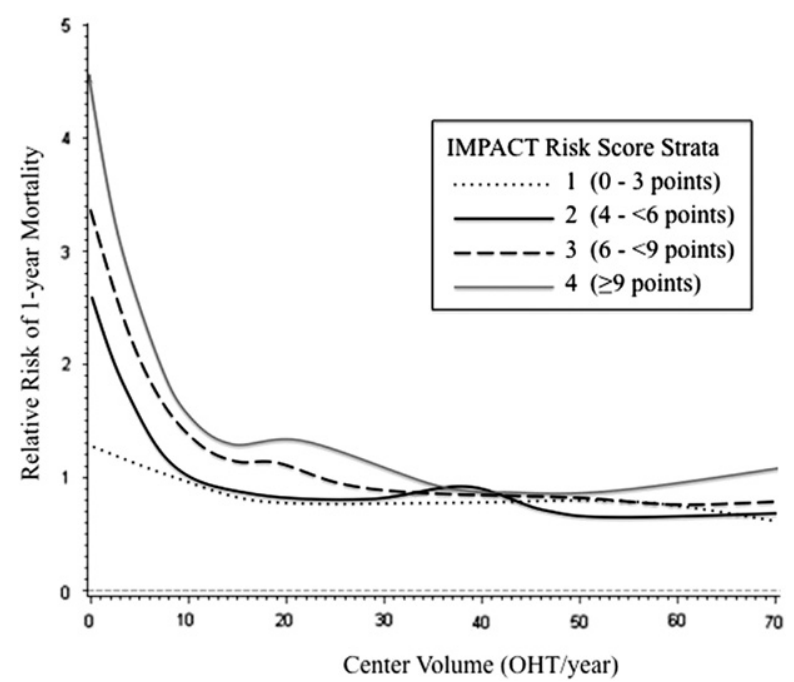

FIGURE 3. Scatter plot of center-level 1-year mortality according to 3 tiers of center volume.

significant survival differences persisted $(P<.001$; Figure 5, $B)$. In this high-risk subgroup, post hoc testing revealed a trend toward a difference in 1-year survival between high-volume and medium-volume centers $(P=.056)$.

\section{Postoperative Complications}

Commonly encountered postoperative complications, according to center volume groups, are shown at the bottom of Table 1. Rates of cardiac reoperation and postoperative

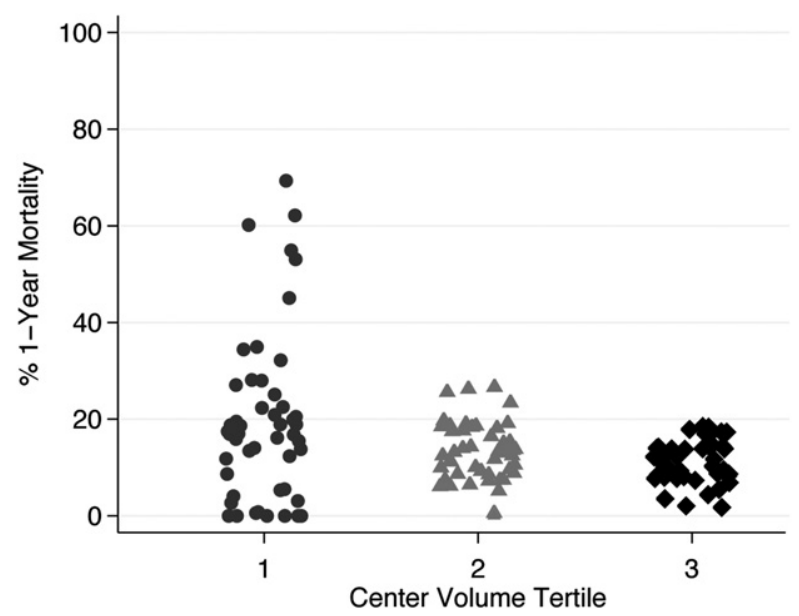

FIGURE 4. Relative risk of 1-year mortality plotted against center volume as a continuous variable on the abscissa. Stratification was performed according to 4 quartiles of recipient risk according to the Index for Mortality Prediction After Cardiac Transplantation (IMPACT) risk index. Relative risk crosses 1 for the lower 2 quartiles at an annual center volume of approximately 10 orthotopic heart transplant $(O H T)$ procedures/y, whereas for the 2 higher quartiles of recipient risk, the risk of 1-year mortality crosses 1 at an annual center volume of approximately 35 to 40 orthotopic heart transplant procedures/y. stroke were similar among the 3 groups. New-onset dialysis, however, was more common at low-volume and medium-volume centers (low, 9.9\%; medium, 8.1\%; high, $7.2 \% ; P<.01)$. In addition, drug-treated rejection in the first year after transplant was more prevalent among low-volume and medium-volume centers (low, $40.3 \%$; medium, $34.4 \%$; high, $28.8 \% ; P<.01$ ).

\section{DISCUSSION}

We used UNOS data to evaluate the effect of recipient risk (as assessed by the IMPACT risk score) on short-term post-OHT mortality across different levels of transplant center volume. Since 2000, in the United States most OHT procedures have been performed at high-volume centers $(64 \%)$, in stark contrast with low-volume centers $(6 \%)$. Accounting for recipient risk, we observed improved 1-year survivals among higher-volume centers, improvements that were most pronounced in the highest quartile of recipient risk. After incorporating tests of interaction in multivariable analysis, institutional volume modified the effect between recipient risk and mortality.

As recipient risk increased, decreasing center volume amplified the risk of early mortality after OHT. This amplification of risk was most pronounced in the range of recipient scores from 10 to 20 . Beyond a risk score of 20, the best-fit lines for observed mortality migrated back within the bounds of the $95 \%$ CI estimates for predicted mortality, likely related to outliers and small numbers of patients in extreme risk categories (Figure 2). In fact, fewer than 1\% of all OHT procedures in this study were performed on OHT recipients with scores greater than 20, and it is therefore difficult to draw meaningful conclusions for this subset of patients. Nonetheless, it is clear that high-risk patients survived with greater frequency than predicted by the IMPACT model at higher-volume centers; conversely, they died with greater frequency than predicted at low-volume centers.

There were several differences in baseline characteristics among the 3 groups of center volume, with high-volume centers tending to OHT recipients with advanced age, more frequent diabetes mellitus, worse renal function, and more frequent use of intra-aortic balloon pumps. Although centers varied in these individual characteristics, it is important to emphasize that overall risk as assessed by the composite risk index was equivalent in the 3 groups. High-volume centers tended to transplant organs with slightly longer ischemic times, and this factor was adjusted for in the multivariable analysis. Low-volume centers were more likely to perform OHT on African American recipients, patients with congenital heart disease, and patients with higher bilirubin levels. Because of the size of the database, several of these statistically significant differences may not have clinical relevance. Congenital heart disease can be a risk factor for adverse outcomes after OHT, 

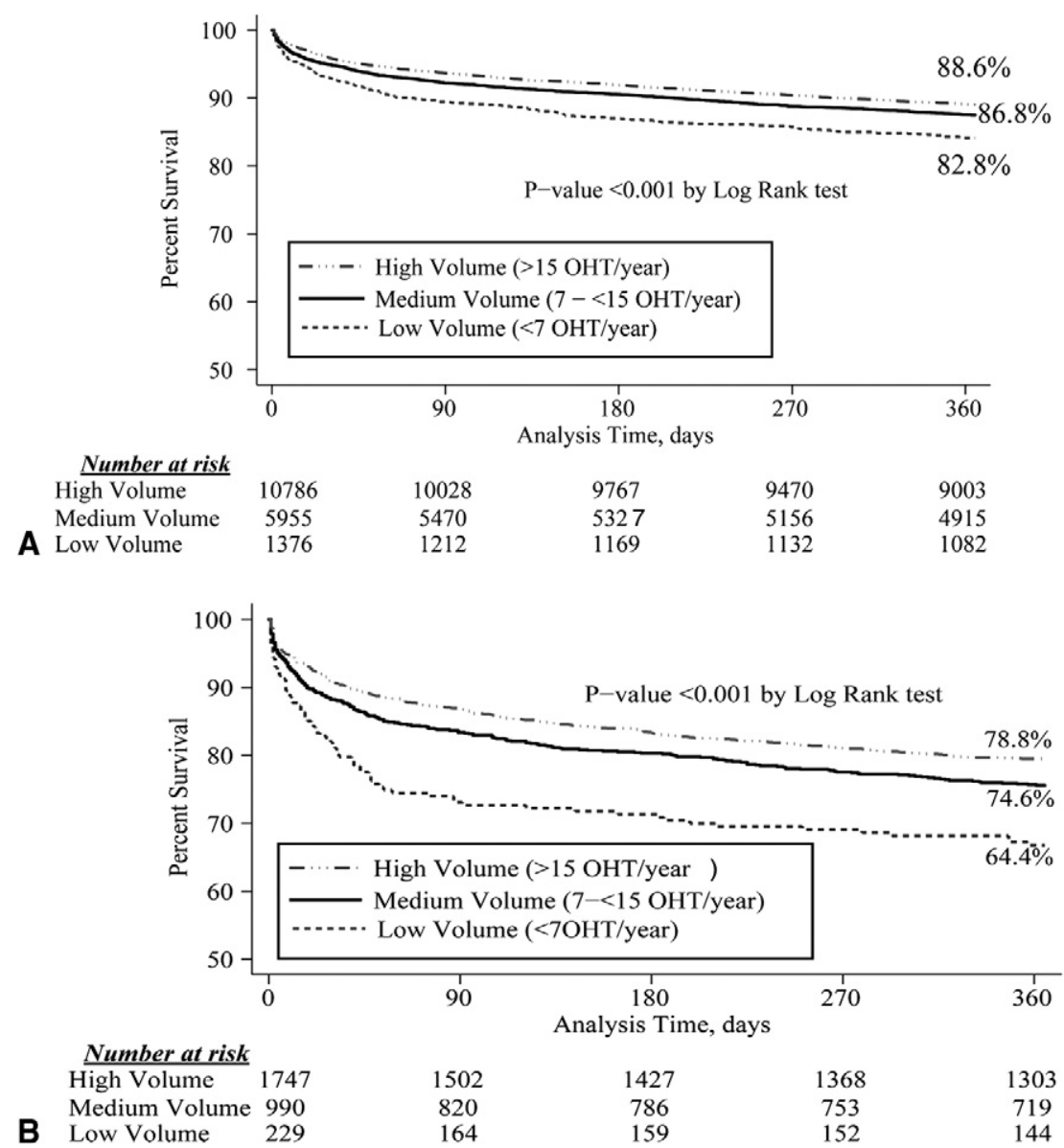

FIGURE 5. A, Kaplan-Meier cumulative 1-year survival of recipients in the cohort overall, stratified by center volume. B, Kaplan-Meier cumulative 1-year survival of high-risk recipients (risk score $>10$ ), stratified by center volume. $O H T$, Orthotopic heart transplant.

however, and the prevalence of congenital heart disease was 3 -fold higher at low-volume centers. We therefore performed a sensitivity analysis that excluded patients with congenital heart disease from the entire analysis. The results remained the same as with congenital heart disease included, suggesting that this variable was not confounding the results.

Analysis of postoperative complications may partially explain the observed survival differences. Postoperative stroke and cardiac reoperation rates were equivalent in the 3 center volume groups. Drug-treated rejection in the first year after OHT was more common in low-volume and medium-volume centers than in high-volume centers, however, with an absolute difference between low-volume and high-volume centers of $12.5 \%$. Although it is difficult to determine from the UNOS database, these differences in drug-treated rejection rates may have been due to inadequately delivered immunosuppression, with the consequence of more rejection-related deaths. Furthermore, postoperative dialysis was more common in low-volume and medium-volume centers. The independent effect of postoperative renal dysfunction on mortality in cardiac surgical patients is well known. ${ }^{17}$ The UNOS variables for these postoperative complications are not reliably coded in the UNOS database, and thus caution is warranted in generating strong conclusions from these data fields.

There are numerous causes of death reported in the UNOS database, with the most common being multisystem organ failure, primary graft failure or cardiac arrest, bacterial septicemia, and respiratory failure. Nearly $20 \%$ of this cohort was missing cause of death information, however, thus preventing reliable use of this variable. Nevertheless, we speculate that the higher rates of dialysis and rejection likely contributed to the differences in observed 30-day and 1-year mortalities. In addition, there were significant differences in 30-day mortality among the different tertiles of center volume, and this suggests that early perioperative deaths may in part account for the observed differences in 1 -year mortality.

\section{Processes of Care}

The principal aim of this study was to achieve a better understanding of the way in which heart transplant centers interact with OHT recipients across the spectrum of recipient 
risk at the national level. These findings should not necessarily be used to impugn low-volume centers, because there are certain low-volume centers that achieve excellent outcomes across the spectrum of recipient risk. Although individual surgeon and provider experience may plausibly account for these results, this issue is clearly quite complex, and these data likely underscore variability in processes of care that may be inherently different across different tiers of center volume. Recent studies have demonstrated that dedicated intensive care unit staff, development of patient safety measures, and integration of critical care pathways may significantly affect outcomes. ${ }^{8,18,19}$ The results of this study should foster further inquiry into systems-based processes of care that may account for these differences in early survival.

Although the specific drivers of the volume effect remain undefined, these data strongly suggest that high-risk OHT recipients are best treated at higher-volume centers. Before advocating such a position, however, it is important to consider risks associated with such referral patterns. For example, having a high-risk patient travel a greater distance to undergo OHT at a high-volume center may affect postOHT compliance, clinic visits, and routine surveillance, not to mention the obvious risks incurred from physical transport of a critically ill patient. Furthermore, from a societal standpoint, transport costs for both recipients and organs may nullify any potential benefits of regionalization. Further studies are necessary to examine the specific processes of care that impart improved outcomes at highervolume centers. Ideally, such studies could elucidate the most important factors present at higher-volume centers, and attempts could be made to incorporate them into lowvolume centers. Ongoing monitoring of outcomes and access to OHT is also paramount to determine whether this center effect persists and to ensure that patients are not denied OHT because of an inability to access a higher-volume center.

With these caveats, however, these data begin to shed light on why higher-volume centers attain superior results. We now know that it is specifically because they succeed with high-risk patients. These are the patients for whom low-volume centers tend not to achieve equivalent results. As a result, we support a paradigm shift toward performing OHT on high-risk patients at higher-volume centers exclusively.

\section{Previous Work}

Although the relationship between hospital volume and outcomes for surgical procedures was initially described in the general surgical population, these associations have been applied to cardiac surgical patients. ${ }^{1,20,21}$ Previous studies have identified this relationship specifically in the context of cardiac transplant as well. ${ }^{12,14,15}$ Although one cannot prove causation in a retrospective study, these studies were performed with a nationwide data set. Hosenpud and colleagues ${ }^{15}$ conducted the first large registry study with UNOS data in OHT during the years 1987 through 1991, and perioperative care has evolved since that time. They reported a $33 \%$ increase in the risk of 1 -year mortality among centers performing fewer than 9 OHT procedures/y. Our group has examined the effect of center volume on post-OHT mortality in a more contemporary cohort (1999-2006) and found that centers performing fewer than $01 \mathrm{OHT}$ procedures/y had a $35 \%$ increase in the hazard of 1-year mortality. ${ }^{12}$ Although recipient variables were incorporated into a multivariable analysis, that study was limited in its ability to discriminate the effect of recipient risk and center volume on short-term mortality. More recently, Russo and colleagues ${ }^{14}$ investigated recipient risk and center volume in the context of post-OHT mortality with UNOS data. ${ }^{14}$ In addition to corroborating these previous observations, we believe that our current analysis expands on these earlier studies in several important ways.

Our current study not only corroborates previous findings but also expands our understanding of this issue. Specifically, there has previously been no standardized recipient risk index for predicting short-term mortality in cardiac transplant. The study by Russo and colleagues ${ }^{14}$ incorporated recipient risk, but that study did not derive a composite risk score in a test group and validate the findings in a separate cohort. Without a composite index, it is difficult to show generalizability from analysis to analysis. Standardization of risk is important as we attempt to analyze which factors lead to improved outcomes for high-risk patients at higher-volume centers. The 12 variables contributing to the 50-point IMPACT risk score (Table E1) represent those most significantly affecting 1 -year mortality for first-time OHT recipients. Furthermore, because of the rigorous statistical methodologies used to derive the risk score, we believe that our findings are robust and accurately reflect how an individual OHT recipient's risk profile affects the risk of early mortality at different levels of center volume. Additionally, although interaction effects have been examined in liver transplants, this is the first study of cardiac transplants to incorporate interaction terms to assess the varying effects of recipient risk across different strata of center volume. $^{22}$

Furthermore, the assignment of center volume for OHT has varied in the literature. Russo and colleagues ${ }^{14}$ defined high-volume as greater than 47 OHT procedures/y, whereas the Center for Medicare and Medicaid Services currently stipulates that centers perform at least 10 OHT procedures/y to maintain certification. ${ }^{23}$ To assign center volume tertiles, we considered each center (with its respective average annual volume) as a single observation, rather than considering each patient as an individual observation. We believe that deriving cut points as was done in this study more accurately reflects a center's annual OHT experience. 
This avoids cut point biases with cut points that are skewed in the upward direction because of a greater overall number of patients undergoing OHT at higher-volume centers. In our analysis, only 6 centers performed more than 47 OHT procedures/y, which is too few to be performing all the high-risk OHT procedures in the United States.

We also treated center volume as a continuous variable. A visual inspection of relative risk for 1-year mortality suggests that the optimal cut point for low-risk OHT recipients may be a center volume around 10 OHT procedures/y. Higher-volume centers performing approximately 35 OHT procedures/y may be best suited to handle the highest-risk recipients. There are only 9 centers that average at least 35 OHT procedures/y, however, and although these data support regionalization of high-risk recipients, 9 centers may be too few to accommodate all high-risk OHT recipients in the United States. Improved means for identifying lower-volume centers that achieve excellent outcomes for high-risk recipients will ensure continued access to OHT for the most critically ill patients awaiting cardiac transplants.

\section{Limitations}

Because of the retrospective nature of this study design, there are certain important limitations. Large national clinical registry studies inherently assume accurate coding of data. It is impossible to determine coding errors, although we have assumed that errors of this nature are likely to be distributed randomly throughout the data set. If this is not the case, these potential errors may have introduced bias in our results. An added limitation of this study is the inability to make strong conclusions about outcomes at centers with very low volumes. Even though the UNOS registry represents a large sample size, centers that perform on average fewer than 1 OHT procedure/y will have variable outcomes. In the absence of more low-volume centers to examine, it is difficult to ascertain whether high mortalities at low-volume centers represent clear trends or natural variability. Additionally, despite the large patient cohort, the event rate of 1-year mortality was low among low-risk recipients. Thus this study may have been insufficiently powered to detect a true survival difference according to center volume among low-risk recipients.

These findings may not fully account for the interaction between donor characteristics and recipient risk. We incorporated donor age and allograft ischemic time, because these variables were significantly associated with postoperative mortality on univariate analysis. When combined in our risk-adjusted multivariable model, the test of interaction between recipient risk and center volume remained significant, suggesting an effect related to center volume independent of these donor characteristics. In light of the limited donor information in the UNOS registry, there may be donor selection factors affecting mortality that vary by center or organ procurement organization, which we have not been able to consider.

Furthermore, the UNOS database includes limited information regarding immunosuppression management, type of rejection, processes of care, and postoperative complications. The strength of the UNOS data set is derived from its relatively complete mortality information. The focus of this study was therefore on 1-year mortality, with reporting of various postoperative complications to supplement the mortality findings. Additionally, retrospective studies cannot control for unrecognized differences in recipient characteristics. We attempted to account for these potential differences by incorporating a validated recipient risk index; however, there may be other variables not accounted for in our risk index that affect short-term mortality. In developing scoring systems, validation is a vital feature. In our original score determinations, we used cross-validation by deriving the score from a random subset and using the remainder of the sample for validation. This methodology is unlikely to have biased the validation; however, we acknowledge that our recipient index will benefit from external validation in an independent sample.

\section{CONCLUSIONS}

In an analysis with UNOS data and our novel recipient risk index, institutional volume acted as an effect modifier on the association between risk and mortality. High-risk patients had higher mortality when undergoing OHT at lowvolume centers and lower mortality when undergoing OHT at higher-volume centers. These differences dissipated among lower-risk recipients. These data support performing OHT on high-risk recipients at higher-volume centers.

\section{References}

1. Luft HS, Bunker JP, Enthoven AC. Should operations be regionalized? The empirical relation between surgical volume and mortality. N Engl J Med. 1979;301: 1364-9.

2. Gordon TA, Burleyson GP, Tielsch JM, Cameron JL. The effects of regionalization on cost and outcome for one general high-risk surgical procedure. Ann Surg. 1995;221:43-9.

3. Meguid RA, Weiss ES, Chang DC, Brock MV, Yang SC. The effect of volume on esophageal cancer resections: what constitutes acceptable resection volumes for centers of excellence? J Thorac Cardiovasc Surg. 2009;137:23-9.

4. Nguyen NT, Paya M, Stevens CM, Mavandadi S, Zainabadi K, Wilson SE. The relationship between hospital volume and outcome in bariatric surgery at academic medical centers. Ann Surg. 2004;240:586-94.

5. Finlayson EV, Goodney PP, Birkmeyer JD. Hospital volume and operative mortality in cancer surgery: a national study. Arch Surg. 2003;138:721-6.

6. Harmon JW, Tang DG, Gordon TA, Bowman HM, Choti MA, Kaufman HS, et al. Hospital volume can serve as a surrogate for surgeon volume for achieving excellent outcomes in colorectal resection. Ann Surg. 1999;230:404-3.

7. Lieberman MD, Kilburn H, Lindsey M, Brennan MF. Relation of perioperative deaths to hospital volume among patients undergoing pancreatic resection for malignancy. Ann Surg. 1995;222:638-45.

8. Dimick JB, Cattaneo SM, Lipsett PA, Pronovost PJ, Heitmiller RF. Hospital volume is related to clinical and economic outcomes of esophageal resection in Maryland. Ann Thorac Surg. 2001;72:334-41.

9. Diaz-Montes TP, Zahurak ML, Giuntoli RL 2nd, Gardner GJ, Bristow RE. Uterine cancer in Maryland: impact of surgeon case volume and other prognostic factors on short-term mortality. Gynecol Oncol. 2006;103:1043-7. 
10. Billingsley KG, Morris AM, Dominitz JA, Matthews B, Dobie S, Barlow W, et al. Surgeon and hospital characteristics as predictors of major adverse outcomes following colon cancer surgery: understanding the volume-outcome relationship. Arch Surg. 2007;142:23-32.

11. Axelrod DA, Guidinger MK, McCullough KP, Leichtman AB, Punch JD, Merion RM. Association of center volume with outcome after liver and kidney transplantation. Am J Transplant. 2004;4:920-7.

12. Weiss ES, Meguid RA, Patel ND, Russell SD, Shah AS, Baumgartner WA, et al Increased mortality at low-volume orthotopic heart transplantation centers: should current standards change? Ann Thorac Surg. 2008;86:1250-60.

13. Weiss ES, Allen JG, Meguid RA, Patel ND, Merlo CA, Orens JB, et al. The impact of center volume on survival in lung transplantation: an analysis of more than 10,000 cases. Ann Thorac Surg. 2009;88:1062-70.

14. Russo MJ, Iribarne A, Easterwood R, Ibrahimiye AN, Davies R, Hong KN, et al. Post-heart transplant survival is inferior at low-volume centers across all risk strata. Circulation. 2010;122(11 Suppl):S85-91.

15. Hosenpud JD, Breen TJ, Edwards EB, Daily OP, Hunsicker LG. The effect of transplant center volume on cardiac transplant outcome. A report of the United Network for Organ Sharing Scientific Registry. JAMA. 1994;271:1844-9.

16. Weiss ES, Allen JG, Arnaoutakis GJ, George TG, Russell DA, Shah AS, et al. Creation of a quantitative recipient risk index for mortality prediction after cardiac transplantation (IMPACT). Ann Thorac Surg. 2011;92:914-2.

17. Chertow GM, Levy EM, Hammermeister KE, Grover F, Daley J. Independent association between acute renal failure and mortality following cardiac surgery. Am J Med. 1998;104:343-8.

18. Zehr KJ, Dawson PB, Yang SC, Heitmiller RF. Standardized clinical care pathways for major thoracic cases reduce hospital costs. Ann Thorac Surg. 1998;66: 914-9.

19. Makary MA, Sexton JB, Freischlag JA, Millman EA, Pryor D, Holzmueller C, et al. Patient safety in surgery. Ann Surg. 2006;243:628-35.

20. Sollano JA, Gelijns AC, Moskowitz AJ, Heitjan DF, Cullinane S, Saha T, et al. Volume-outcome relationships in cardiovascular operations: New York State, 1990-1995. J Thorac Cardiovasc Surg. 1999;117:419-30.

21. Peterson ED, Coombs LP, DeLong ER, Haan CK, Ferguson TB. Procedural volume as a marker of quality for CABG surgery. JAMA. 2004;291:195-201.

22. Segev DL, Kucirka LM, Nguyen GC, Cameron AM, Locke JE, Simpkins CE, et al. Effect modification in liver allografts with prolonged cold ischemic time. Am J Transplant. 2008;8:658-66.

23. The Center for Medicare and Medicaid Services Medicare program; hospital conditions of participation: requirements for approval and re-approval of transplant centers to perform organ transplants. Final rule. Fed Regist. 2007;72:15197-280.

\section{Discussion}

Dr R. Duane Davis (Durham, NC). First, I congratulate you on an excellent presentation. This is not a shocking finding, in a team sport like heart transplant, that better outcomes are associated with higher volumes. I want to focus a little bit more on how you defined what was a large-volume versus a small-volume center. There was a recent report in the Journal that used essentially the same UNOS data set that you did. They divided it into quartiles; in fact, the low volume was approximately 15 and less, and then marched it up, with the high-volume centers approximately 34 and over. For each of the groups, there was a reduction in the actual mortality risk. You have added recipient risk, as quantified by your IMPACT score, to the analysis. High-risk recipients would seem to do better in centers that are doing higher volumes of procedures. Did your analysis actually look at where the volume threshold effect was? Did it continue to decline as the volumes increased? If you look at the International Society for Heart and Lung Transplantation registry, which puts out the slide every year associating recipient mortality with center volume, the place where it plateaus is approximately 35 to 40 . I would assume that that would be similar in this analysis, but you have tended to focus more on just the very small versus moderate sized transplant programs.
Dr Arnaoutakis. Thank you, Dr Davis, for your kind comments and important points and questions. With respect to our stratification methods, we counted each center as a single observation when we made our stratifications. We have done our center volume analyses in this fashion in the past, and we believe that it is actually very important to do it in this fashion. When we divide into tertiles according to defining average volume in this fashion, there is roughly a third, or approximately 47 centers, that are above 15 heart transplants per year. When each individual patient is counted as a single observation, that significantly skews the cut points in the upward direction. If we were then to define centers according to those cut points, we would actually limit significantly the number of centers defined as high volume for the purposes of this study. So that was why we defined them as such.

In our analysis, we did perform both continuous and categoric, or dichotomous, cut points for center volume. Because we incorporated interaction terms, it becomes increasingly complicated and difficult to explain simply the effect modification when examining the interaction among multiple continuous variables. With regard to your point about the International Society for Heart and Lung Transplantation registry, however, we did examine that in a continuous fashion, with spline terms as well as graphically inspecting Lowes S curves, and it looks to us, especially for the high-risk recipients derived from UNOS data, which obviously varies somewhat from the international registry, that there does seem to be a plateau that occurs between 20 to 30 procedures per year, which still limits the number of centers although not as much so as when we make cut points above 45 , where then we are only talking about a handful of centers, which may limit access to care for some patients who are not near these centers.

Dr Davis. The next component of this is that we shouldn't really be in the business of trying to shut down programs. It should be in the idea of how do we improve the quality of all the programs. So how can we essentially raise the boat? And are there things, and this is something that you are not going to get out of the UNOS data set but you can make some assumptions, that are necessary to be able to do this well? And what can we learn from this type of data set analysis to say, okay, if you are going to be in the business of heart transplant, these are the critical items that you need to be doing if you are going to be really successful? Again, my congratulations on an excellent presentation.

Dr Arnaoutakis. Thank you again. Your point is absolutely right and well taken. The study intention and these findings are not meant to impugn centers of any volume whatsoever. If you look closely, there are several centers in the low-volume category that achieve excellent outcomes. And so you are getting at a very important point, that these findings don't prove any causation but show associations. What we need to pursue from here on is to identify the processes of care that we believe are intimately involved with these findings. One way to do this is to go back to institutional series, where we have access to variables that are not included in the UNOS database, which although it has many variables does not encompass all variables. For example, we have looked in our institutional series at the distance that patients live from the transplant center and have found an association with greater rates of rejection. So there may be some follow-up study that can be performed at individual centers both in the high-volume end of 
the spectrum and in the low-volume end of the spectrum to identify these processes of care.

It is difficult with the UNOS database to glean any information regarding independent surgeon experience and surgeon volume, which may play some role. As well, perioperative care may vary across the spectrum of different centers with different volumes, and we believe that this is also intimately involved with these findings. And so some of the pathways and structures that are set up at various intensive care units may shed more light on these differences.

We do believe that these findings begin to shed light on the outcome differences that we have observed, in that the differences seem to be dictated in large part by outcomes of recipients who are at the highest end of the risk spectrum.

Dr Craig R. Smith (New York, NY). Very briefly, did you consider the possibility that a low-volume center that had been in business for a long time might be okay, which you might see by looking at total historical transplant volume or total number of survivors?

Dr Arnaoutakis. We didn't specifically look at that. We did look over time, and it is difficult to tell whether a center has gone from 1 to 0 transplants per year if that center remains open. We looked at the patterns, and we made assumptions according to whether they had closed temporarily and then reopened. We did incorporate time into our models, but not with the specific question that you asked. That is an important point, however, for understanding factors that may play into low-volume centers that achieve excellent outcomes. That is something that we are going to look into further.

Dr Giovanni Battista Luciani (Verona, Italy). I enjoyed your presentation. On one of the first slides, in the lower center volumes there was a higher prevalence of congenital heart disease as an indication for surgery. I was intrigued by that, because it has been recognized that this is an increased risk factor for mortality early and late after transplant. Did you try running your analysis excluding that diagnosis, and would it come out the same? Thank you.

Dr Arnaoutakis. Thank you for pointing that out. We were intrigued by that as well. We didn't expect to see such a magnitude of difference. Congenital heart disease is accounted for in our risk score, and so we actually did not perform the analysis excluding those patients, because we considered that it was already adjusted for. So I don't believe that that would be driving any of these findings, but it is an important thing that we could examine. 
TABLE E1. Description of Index for Mortality Predication After Cardiac Transplantation (IMPACT) risk score and allocation of points by variable

\begin{tabular}{|c|c|}
\hline Covariates & $\begin{array}{c}\text { Points } \\
\text { assigned }\end{array}$ \\
\hline Age $>60 y$ & 3 \\
\hline \multicolumn{2}{|l|}{ Bilirubin (serum, mg/dL) } \\
\hline $0-0.99$ & 0 \\
\hline $1-1.99$ & 1 \\
\hline $2-3.99$ & 3 \\
\hline$\geq 4$ & 4 \\
\hline \multicolumn{2}{|l|}{ Creatinine clearance $(\mathrm{mL} / \mathrm{min})$} \\
\hline$>50$ & 0 \\
\hline $30-49$ & 2 \\
\hline$<30$ & 5 \\
\hline Dialysis between listing and transplant & 4 \\
\hline Female sex & 3 \\
\hline \multicolumn{2}{|l|}{ Heart failure etiology } \\
\hline Idiopathic & 0 \\
\hline Ischemic & 2 \\
\hline Congenital & 5 \\
\hline Other & 1 \\
\hline Infection & 3 \\
\hline Intra-aortic balloon pump & 3 \\
\hline Mechanical ventilation before transplant & 5 \\
\hline \multicolumn{2}{|l|}{ Ethnicity } \\
\hline White & 0 \\
\hline African American & 3 \\
\hline Hispanic & 0 \\
\hline Other & 0 \\
\hline Temporary circulatory support (ECMO/extracorporeal VAD) & 7 \\
\hline Older-generation pulsatile VAD & 3 \\
\hline New-generation continuous VAD (excluding HeartMate II) & 5 \\
\hline HeartMate II & 0 \\
\hline Total points possible & 50 \\
\hline
\end{tabular}

